\title{
The Energetic Particle Populations of the Distant Heliosphere
}

\author{
F. B. McDonald*, A. C. Cummings ${ }^{\dagger}$, E. C. Stone ${ }^{\dagger}$, B. C. Heikkila ${ }^{\ddagger}$, N. Lal ${ }^{\ddagger}$, and \\ W. R. Webber ${ }^{\text {II }}$ \\ *Institute for Physical Science and Technology, University of Maryland, College Park, MD 20742, USA \\ ${ }^{\dagger}$ California Institute of Technology, Pasadena, California 91125, USA \\ ${ }^{*}$ NASA/Goddard Space Flight Center, Greenbelt, MD 20771, USA

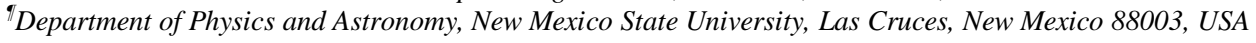

\begin{abstract}
In late 2003 as Voyager 1 moves beyond $90 \mathrm{AU}$ in the recovery phase of solar cycle 23, the effects of the termination shock (TS) and the heliosheath on particle transport are becoming more evident. There are now detectable fluxes of $2.5-70 \mathrm{MeV}$ electrons but at low intensity levels that suggest they are heavily modulated in the heliosheath. The modulation of galactic and anomalous cosmic rays is significant but much smaller than observed at $1 \mathrm{AU}$. At V1 a large increase of $\mathrm{MeV}$ ions was observed with a flat energy spectra which persisted over a period of 6.5 months. A second event has now been in progress for some eight months. These ions appear to originate at the TS. At V2, 17 AU behind V1, there are a series of 8 increases of low energy ions that occur approximately every 140 days starting in late 2000. Many of these increases can be related to specific periods of high solar activity that occurred some 6 months earlier.
\end{abstract}

\section{INTRODUCTION}

The large, complex structure of the outer heliosphere is created by the interaction of the outward flowing supersonic solar wind with the local interstellar medium (LISM). The suddenly heated and decelerated solar wind forms the heliosheath, which is expected to have an extended heliotail produced by the relative motion of the heliosphere with respect to the LISM (Fig. 1) $[1,2]$.

Galactic cosmic rays (GCR) traverse the heliosheath and cross the termination shock (TS) of the solar wind before interacting with the supersonic solar wind. They may experience a modest local reacceleration through their encounters with the TS [3,4]. At times of low to moderate solar activity, anomalous cosmic rays (ACR) are the dominant energetic particle components below 50 - $60 \mathrm{MeV} / \mathrm{n}$. These predominantly singly-charged [5] ions are assumed to be accelerated at the TS [6] but may undergo significant pre-acceleration in the solar wind [7]. With their high rigidity and relatively low velocity they are especially sensitive to the various modulation processes. At times of higher solar activity there are increases of low energy ions associated with the passage of large interplanetary disturbances known as Global Merged Interaction Regions (GMIR), which are formed through the coalescence of high-speed solar wind streams and multiple interplanetary coronal mass ejections. These low energy solar/interplanetary ions may experience further acceleration as the associated GMIR sweeps across the TS [8]. 


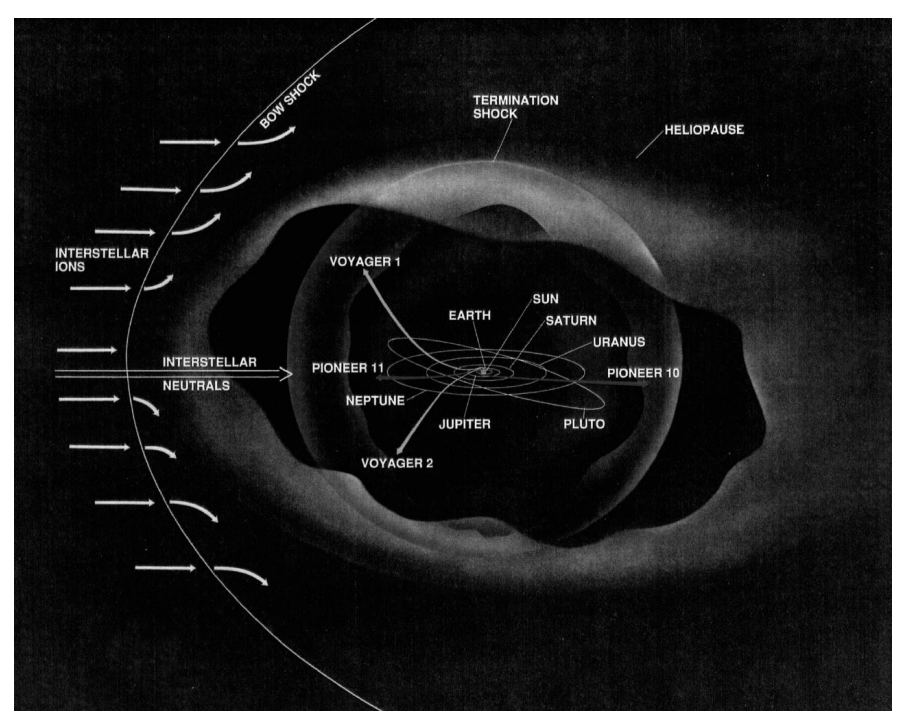

Figure 1. Schematic view of the heliosphere.

Over the past 32 years the cosmic ray experiments on the IMP at $1 \mathrm{AU}$, and on Pioneer 10 (out to 65 AU through 5/96) and Voyagers 1 and 2 at ever increasing heliocentric distances have constituted a unique network for observing the temporal and spatial variations of the different energetic particle populations. In late $2003 \mathrm{~V} 1$ was just beyond $90 \mathrm{AU}$ at a heliolatitude $\lambda=34^{\circ} \mathrm{N}$ while V2 approached $72 \mathrm{AU}$ at $\lambda=24.7^{\circ} \mathrm{S}$ (Fig. 2). At this time the reversal of the solar dipole moment following this past solar maximum was complete and the heliosphere was in a $\mathrm{qA}<0$

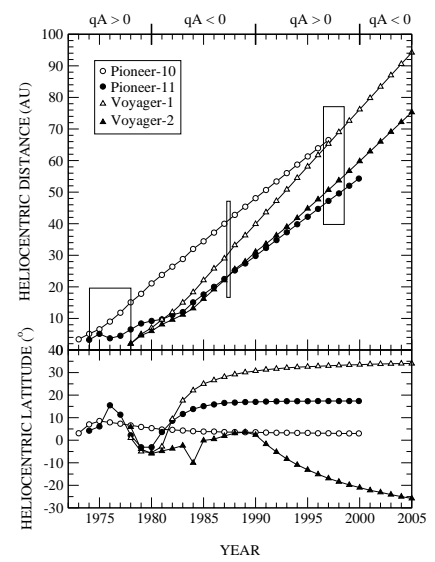

Figure 2. Pioneer 10,11 and Voyager 1, 2 trajectory information. The rectangles are the times of peak cosmic ray intensity over the solar minimum of cycles 20,21 and 22. 
state. At $1 \mathrm{AU}$ the onset of recovery of galactic cosmic rays following solar maximum was different from that observed by neutron monitors over the previous 4 cycles. A similar plateau feature was also seen in the GCR recovery at V2. At V1 there were two significant increases of $2.5 \mathrm{MeV}$ ions with the first beginning in 2002.54 and lasting some 6.5 months and the $2^{\text {nd }}$, which began in mid-August 2003 and continued at least until 1 April 2004. At the same time there were increases in $2.5-70 \mathrm{MeV}$ electrons, $30-56 \mathrm{MeV}$ ACR He, and $70-300 \mathrm{MeV}$ galactic cosmic rays at V1 that were not observed at V2. At V2 the $2.5 \mathrm{MeV}$ ion events also had a different intensity level and time history from the V1 events. Clearly by mid 2002.5 V1 had arrived in the close vicinity of the termination shock. In this paper we present an overview of these Voyager observations along with the relevant 1 AU data.

\section{COSMIC RAY MODULATION IN THE DISTANT HELIOSPHERE}

Observations from the heliospheric network, which now span more than 1.5 heliomagnetic cycles, have significantly changed our concepts of cosmic ray modulation. In this section we discuss the effects of cycle 23 through 2004.25 and compare this period with the solar minimum and maximum intensities from previous cycles.

The GCR He (265 MeV/n) data are shown in Fig. 3 (26 day averages) for the period 1996.0 2004.25. At $1 \mathrm{AU}$ around the years of the solar minimum (1997) and solar maximum (2000.6) intensity of $265 \mathrm{MeV} / \mathrm{n}$ He are very close to those of cycle 21, with the intensity decreasing by a factor of 4.5 for each cycle. Following a very small recovery in late 2001, the GCR He intensity at 1 AU exhibits a $\sim 2.5$ year plateau period which is very different from any previous recovery in the neutron monitor era. This behavior is also seen for all 4 components at V2 $(\bar{r}=67 \mathrm{AU})$. However at V1 $(\bar{r}=87 \mathrm{AU})$, the time histories are significantly different as the spacecraft apparently nears the termination shock.

As they travel outward the Voyagers and Pioneer 10 sample a different region of space over each phase of the solar and heliomagnetic cycles. To obtain a unified description of the spatial variations it is necessary to combine the data from multiple cycles. In this initial study we have concentrated on the times of solar minimum and maximum. The solar minima of cycles 20 and 22 with $q A>0$ are very similar in that the Climax neutron monitor counting rates and the IMP $8 \mathrm{H}$ and He spectra were essentially the same in each cycle. Thus the Pioneer and Voyager intensities for these periods can be combined. The radial distribution for the three solar minimum periods for $175 \mathrm{MeV}$ GCR H $(130-220 \mathrm{MeV})$ and $265 \mathrm{MeV} / \mathrm{n}(150-380 \mathrm{MeV}) \mathrm{He}$ shows essentially no change in their intensities between about 12 and 73 AU (Fig. 4). This radial dependence is similar to that of ACR oxygen [25]. The latitudinal gradients are small $\left(0.0 \pm 0.1 \% /^{\circ}\right.$ for 175 GCR H and $0.0 \pm 0.2 \% /^{\circ}$ for $265 \mathrm{MeV} / \mathrm{n}$ GCR He at $61 \mathrm{AU}$ in 1996) [26]. The GCR He intensity for these two qA>0 periods is 0.295 at $1 \mathrm{AU}, 0.43$ at $14 \mathrm{AU}$ and $0.44 / \mathrm{m}^{2}-\mathrm{s}-\mathrm{sr}-\mathrm{MeV} / \mathrm{n}$ at $75 \mathrm{AU}$. The expected value of the interstellar intensity is 1.2 [9]. Based on this interstellar intensity and neglecting drift effects, $85 \%$ of the GCR He cosmic ray modulation at solar minimum occurs at the termination shock and in the heliosheath with only $15 \%$ occurring inside the termination shock. Most of the latter occurs between 1 and 15 AU.

For cycle 21 with $\mathrm{qA}<0$ the radial distribution of GCR He is steeper and suggests larger gradients from $\sim 25 \mathrm{AU}$ out to the TS, again similar to that observed for ACR oxygen. Extrapolating the 1987 data to 100 AU suggests a $44 \%$ increase in intensity for cycle 23 over that of cycle 22 if latitudinal gradients are not important at 100 AU. This increase may partly reflect reacceleration at the $\mathrm{TS}$. 


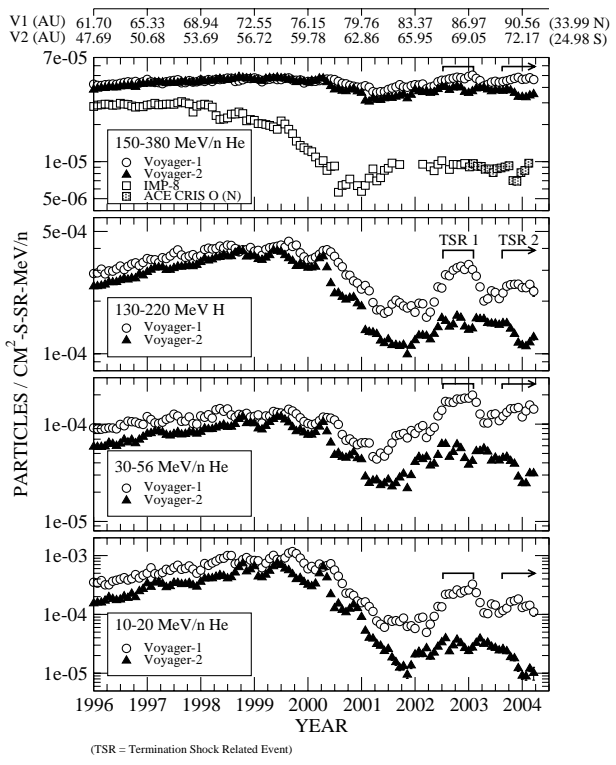

Figure 3. Time histories for GCR He and H and ACR He (26 Day Avg.). There are gaps in the 1 AU GCR He data after 2003 which are filled in by using normalized $122-236 \mathrm{MeV} / \mathrm{n}$ Oxygen data from the ACE CRIS experiment. The time of the termination shock related (TSR) low energy ion increases is shown by brackets.

Conventional 2D simulations of the cosmic ray radial distribution at solar minimum can produce the low radial gradients in the outer heliosphere but are inconsistent with the small latitudinal gradients. For $175 \mathrm{MeV}$ GCR $\mathrm{H}$ with a rigidity of $0.6 \mathrm{GV}$, drift effects should be smaller, but the radial dependence is very similar to that of GCR He. With an interstellar intensity of $23 / \mathrm{m}^{2}$-s-sr-MeV and neglecting drifts, $<10 \%$ of the $175 \mathrm{MeV}$ GCR modulation at solar minimum occurs inside the termination shock. Similar conclusions on significant modulation in the heliosheath were reached earlier by Webber and Lockwood [9].

At $1 \mathrm{AU}$ the solar maximum intensities are virtually identical for cycles 21 and 23, suggesting that modulation conditions throughout the heliosphere at this time were very similar. Normalizing the $1 \mathrm{AU}$ cycle 22 solar maximum intensity to that of cycles 21 and 23 unifies the solar maximum intensities in a remarkable way. The data in Fig. 4 show that the spatial variation of GCR He has the functional form $g_{r}=j^{-1} d j / d r=G_{0} / r$ (where $r$ is the heliocentric distance in AU) with $g_{r}$ having a value of $(73 / r) \% / A U$ for $r>15 \mathrm{AU}$. In the fits to the data, the dimensionless quantity $G_{0}=d(\log j) / d(\log r)$ is assumed to be piece-wise constant. It then follows that the more familiar logarithmic gradient $g_{r}(\% / A U)=d(\log j) / d r=G_{0} / r$ where $G_{0}$ is expressed in percent and $r$ in $\mathrm{AU}$.

This procedure can also be applied to $130-220 \mathrm{MeV}$ GCR $\mathrm{H}$ using a normalizing factor of 1.5 , which is very close to the value required for GCR He (Fig. 3). The organization of the hydrogen data closely resembles that of GCR He with $g_{r}=149 / r \% / A U$. The increasing 


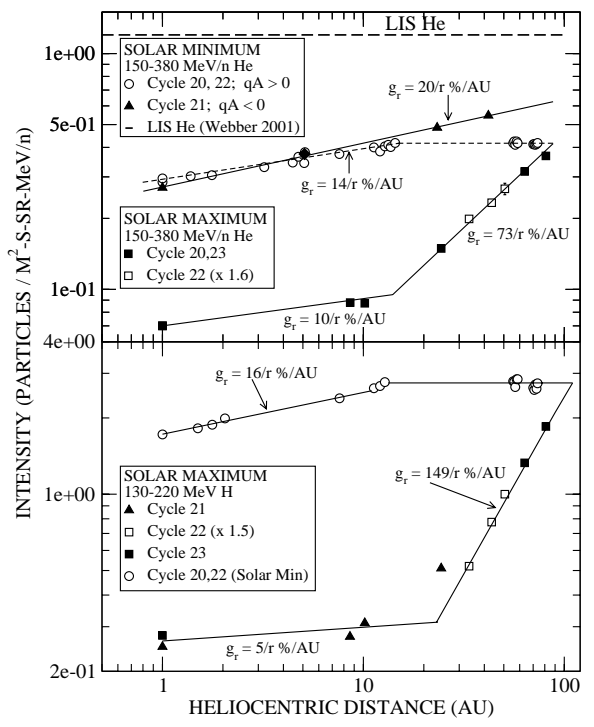

Figure 4. Solar maximum (normalized at $1 \mathrm{AU}$ ) and solar minimum radial intensity distributions for GCR $\mathrm{H}$ and He for cycles 21, 22 and 23. The solid circle at $5 \mathrm{AU}$ in the top panel uses data from the Ulysses KET Experiment [26].

modulation of GCR He (1.5 GV), GCR H $(0.6 \mathrm{GV})$ and electrons $(0.1 \mathrm{GV})$ with decreasing rigidity indicates that the heliosheath modulation is strongly rigidity dependent.

The GCR radial intensity distributions at solar maximum indicate that the changes that produce the 11-year modulation cycle occur mainly in the outer heliosphere between $15 \mathrm{AU}$ and the TS. The extrapolation of the solar maximum data intersects the solar minimum intensity levels at 98.5 AU (GCR He) and 101.5 AU (GCR H). These data suggest that in cycle 23 the heliosheath modulation did not change significantly between solar minimum in 1997-98 and solar maximum in 2001 .

\section{ELECTRONS}

The galactic cosmic ray electron component should consist of directly accelerated primaries, or interstellar secondaries from the decay of charged pions, and at lower energies, knock-on electrons produced by the passage of higher energy cosmic rays through the interstellar medium. At energies below some $200 \mathrm{MeV}$ these electrons are the source of the lower energy diffuse gamma and $\mathrm{x}$-ray emissions from the galaxy and may play a major role in ionizing and heating the interstellar medium. Within the heliosphere they are strongly modulated, even at solar minimum. Between 1 and $10 \mathrm{AU}$ at energies below some $40 \mathrm{MeV}$ there is a large contribution from Jovian electrons. The Voyager missions to the outer heliosphere offer an ideal opportunity to directly observe this component and to study the modulation process at very low rigidities. 
The Voyager CRS experiment (E. C. Stone, P.I.) has two telescope systems that respond to electrons - the High Energy Telescope (HET), 2.5 - $10 \mathrm{MeV}$ e and the Electron Telescope, 6 $160 \mathrm{MeV}$ e [10]. Except for periods when there are large fluxes of electrons of Jovian or solar origin, the response of these telescopes have been dominated by background produced by high energy protons. For example over the 1987 solar minimum at $22 \mathrm{AU}$ all electron channels exhibit a negative latitudinal gradient similar to that obtained for GCR and ACR ions instead of the positive latitudinal gradients expected for electrons in a $q A<0$ epoch. The episodes of strong solar activity over the September, October 1989 and March/June 1991 produced well-defined electron increases at V2 $(\bar{r}=32 \mathrm{AU})$. However these events were much smaller at V1 $(\bar{r}=42 \mathrm{AU})$, indicating a large negative radial gradient.

Over most of cycle 23 all of the V1 and V2 electron channels are at similar background levels. However beginning at 2002.5 at V1 there was an increase in the $2.5-70 \mathrm{MeV}$ e intensity (Fig. 5). There was no corresponding increase in the V2 electron intensity. The V1 increase is associated with the reduced modulation levels of the ACRs and GCRs and with the unusual increase of $2.5 \mathrm{MeV}$ (Fig. 8) ions as discussed in the next section. The high variability of the electron component (Fig. 8) indicates that it is especially sensitive to small changes in the modulation conditions.

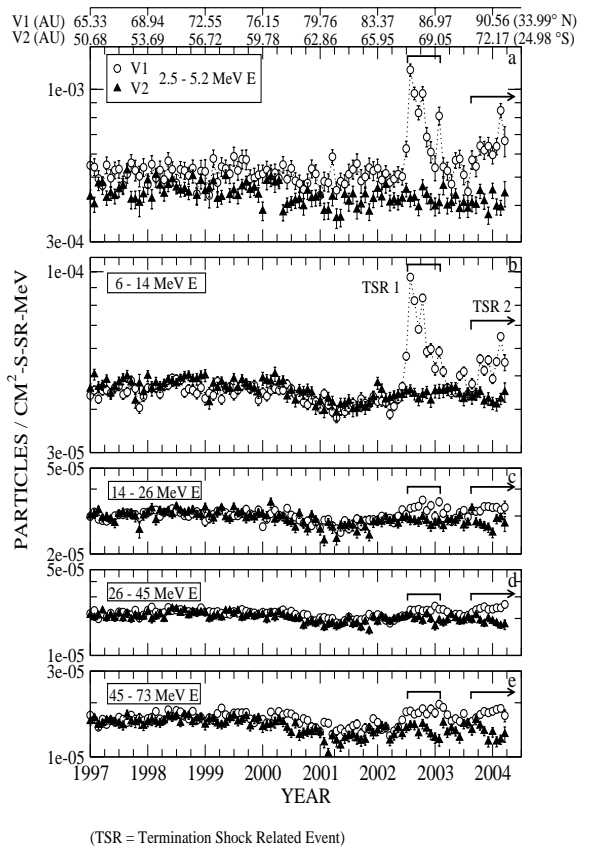

Figure 5. V1 and V2 electron time history (26 Day Avg) 1997-2004.14. 


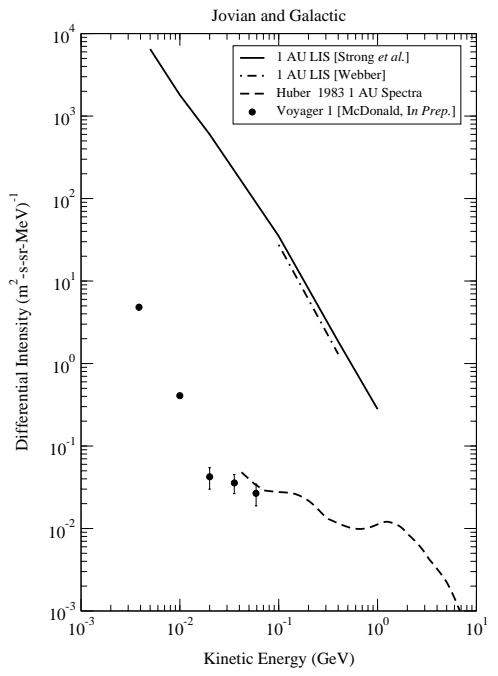

Figure 6. V1 electron spectrum (2002.57 - 2002.85) corrected for background using the detector response function [10]. The local interstellar spectra are those of Webber et al. and Strong et al.. The 1 AU electron data from Huber [24] corresponds approximately to a similar time in the modulation cycle some 19 years earlier.

The V1 electron spectrum for the period 2002.54 - 2002.84 (Fig. 6) is steep at energies below $15 \mathrm{MeV}$ and relatively flat from $15-60 \mathrm{MeV}$. At these higher energies the intensities are similar to the 1983 spectra at 1 AU obtained by Huber [24]. Potgieter and Ferreira have shown there can be significant reacceleration of electrons at the TS [11]. This process may produce the large increase below $20 \mathrm{MeV}$.

Webber et al. [12], using new data on the low-frequency galactic non-thermal radio emission and Strong et al. [13] using the diffusive galactic continuum gamma ray emission from the Comptel experiment on the Compton Gamma Ray Observatory over an energy range of 0.75 $30 \mathrm{MeV}$ have obtained new estimates of the interstellar electron spectra (Fig. 6). The agreement between these 2 very different approaches is quite good. However Strong et al. [14] and Langner et al. [15] have shown that plausible changes in the interstellar parameters can produce large changes in the LIS spectra.

At $20 \mathrm{MeV}$ the electron intensity at V1 is a factor of 3000 below the estimated electron LIS. If the LIS is correct this implies a large modulation of this component in the heliosheath.

\section{ENHANCEMENTS OF MEV IONS NEAR THE TS}

One of the features of the outer heliosphere is the frequent increases in the intensity of low energy ions. We term these increases solar/interplanetary events to distinguish them from the solar energetic particle (SEP) events of the inner heliosphere. Beyond some $10-20$ AU these 


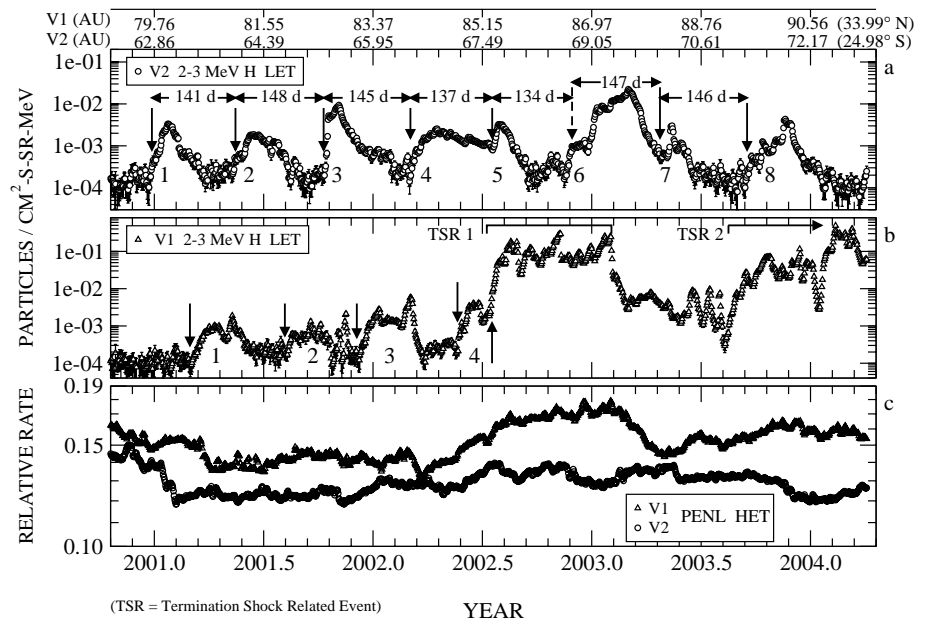

Figure 7. V1, V2 time history of $2.5 \mathrm{MeV}$ ions (5 Day Moving Avg). The arrows in panel "a" are our best estimate of the onset of the low energy events. The Pen $\mathrm{L}$ rate responds to $70 \mathrm{MeV} \leq \mathrm{H} \leq 300 \mathrm{MeV}$ and heavier ions $>70$ $\mathrm{MeV}$. In panel $\mathrm{C}$, the $\mathrm{V} 2$ rate is always below that of $\mathrm{V} 1$.

ions are convected out by large-scale disturbances in the solar wind. For example, they now require some 6 months to reach the present location of V2. It is possible that their composition may have evolved significantly over their six-month transit period through the preferential acceleration of pickup ions [7]. A summary of previous studies of these events in the outer heliosphere can be found in Decker et al. [16].

In mid-2002 as Voyager 1 moved beyond $85 \mathrm{AU}$ there began an unusual increase of $2.5 \mathrm{MeV}$ ions that remained at a level near $0.1 \mathrm{H} / \mathrm{cm}^{2}$-s-sr-MeV for some 6 months (Fig. 7, 8, 9) [17,18]. These V1 intensities are at least 20 times and sometimes 500 times greater than that at V2. Cummings et al. [19] and Krimigis et al. [17] have reported large anisotropies generally directed outwards from the sun along the direction of the magnetic field. The energy spectra has a slope of $\sim-1.5$ at energies below $\sim 1.5 \mathrm{MeV}$ and -2.5 at higher energies. This unusual enhancement must be related to the close proximity of V1 to the termination shock and will be referred to as Termination Shock Related (TSR) event. This first major TSR increase was terminated starting at 2003.15 by the passage of a series of large transient increases in the interplanetary magnetic field.

Starting in 2003.625 there began a second TSR increase that is still in progress at this time. The $2.5 \mathrm{MeV}$ ions in TSR 2 show a greater variability. There are periods when the $2.5 \mathrm{MeV}$ ion intensity is larger in TSR 2 than in the $1^{\text {st }}$ event but the electron and GCR intensities are lower. 


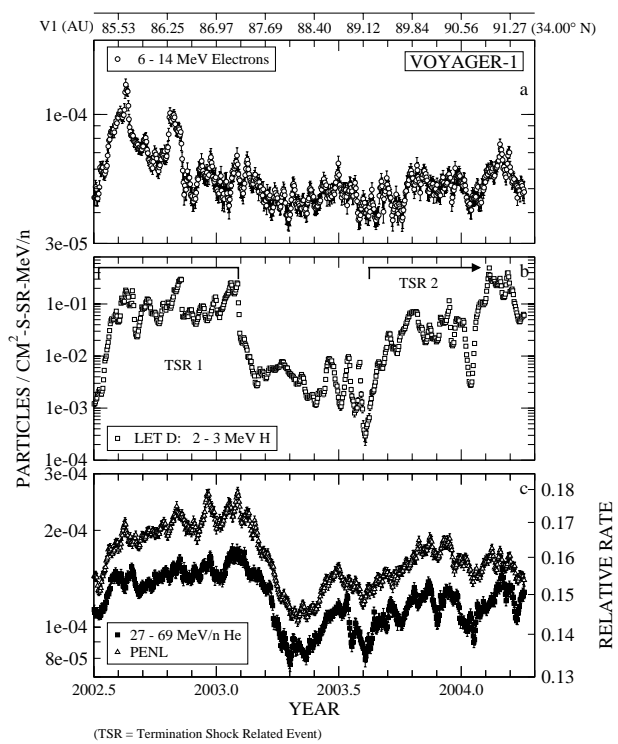

Figure 8. Time histories (5 day moving averages) of Voyager 1 cosmic ray intensities. a) $6-14 \mathrm{MeV}$ electrons, b) $2-3 \mathrm{MeV} \mathrm{H}$, and c) $27-69 \mathrm{MeV} / \mathrm{n} \mathrm{ACR} \mathrm{He}{ }^{+}$(solid squares) and the Pen L relative rate (H: $70<\mathrm{E}<300 \mathrm{MeV}$, and $\mathrm{Z}>1$ ions $\mathrm{E}>70 \mathrm{MeV} / \mathrm{n}$ ) (open triangles). The data is from the Voyager CRS experiments, which consists of 7 multi-element solid-state energetic particle telescopes covering electrons $(2-160 \mathrm{MeV}), \mathrm{H}$ and $\mathrm{He}(1.8-500$ $\mathrm{MeV} / \mathrm{n}$ ) and heavier nuclei through Fe. The $27-69 \mathrm{MeV} / \mathrm{n}$ ACR He is the lower data set in Panel C.

At V2 from 2000.8 - 2003.2 there is a series of well-defined but modest enhancements of $\mathrm{MeV}$ protons that occur at a quasi-periodic rate of $\sim 140$ days and persist on the average for 0.3 years ( 4 solar rotations) (Fig. 7). Six of the events are closely associated with increases of 100 $\mathrm{km} / \mathrm{s}$ in the solar wind speed which can be identified with specific periods of solar activity. For example, the detailed modeling of the $1 \mathrm{AU}$ solar wind and magnetic field data by Wang et al. [20] have shown that event 1 is associated with the solar activity at the time of the large Bastille Day SEP on July 14, 2000. Event 3 appears to be associated with the series of large solar events centered on April 15, 2001. The first four events reach V1 after a delay of some 0.2 years, corresponding to an average transit speed of $420 \mathrm{~km} / \mathrm{s} \mathrm{(Fig.} \mathrm{9).} \mathrm{At} \mathrm{V1} \mathrm{the} \mathrm{intensity} \mathrm{level} \mathrm{of} \mathrm{the}$ first three events was on average $40 \%$ of that at $\mathrm{V} 2$ indicating a mean radial intensity gradient of $-5.5 \%$ per AU. In contrast, the Voyager 1 event beginning in mid-2002 persisted for more than six months with intensities 20 to 500 times greater than at Voyager 2. Such a large disparity has not previously been observed in the outer heliosphere. 


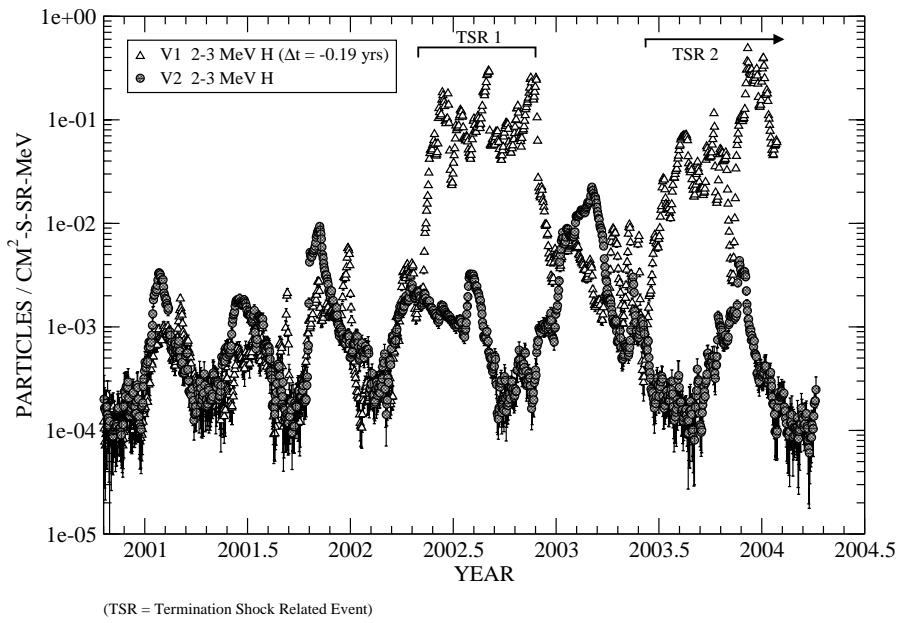

Figure 9. The V1,V2 low energy ion data of Fig. 7 except the V1 data has been displaced by -0.19 years corresponding to the expected solar wind transit time from V1 to V2.

\section{DISCUSSION}

This simultaneous increase of GCRs, ACRs, and $2.5-70 \mathrm{MeV}$ electrons at V1 in mid-2002 is produced by a reduced modulation in the distant heliosphere. Such a recovery is not unexpected at this time some 2 years after the maximum of solar activity in cycle 23 . We suggest that the $2.5-70 \mathrm{MeV}$ electrons are of galactic origin that have been reaccelerated at the termination shock as suggested by Potgieter and Ferreira [11]. We argue that the accompanying increases in ions, TSR 1 and TSR 2, are the type of precursor event that would be expected just as ion increases are seen in the region upstream of the Earth's bow shock.

This interpretation is reinforced by the response at V1 to the transients that produce the 8 energetic particle increases at V2 (Fig. 9). As previously noted, the first 4 of the V2 increases are seen at V1 after a delay of 0.19 years. The onset of TSR 1 and 2 follows the passage of events 4 and 7, respectively. The termination of TSR 1 is associated with the passage of event 6 . The large transient decrease in TSR 2 at 2004.03 coincides exactly with the passage of event 8 when the increased plasma velocity observed at $\mathrm{V} 2$ is taken into account.

The lower GCR, ACR and electron intensity at the time of TSR 2 is probably the result of the TS moving to a larger heliocentric distance as would be expected at this time of declining solar activity and increasing solar wind velocity [21].

The anisotropy upstream of the shock is determined by the requirements that the anisotropy and intensity must be continuous across the shock [3]. This requires that the average radial upstream anisotropy (near the termination shock) equal that downstream, which is determined by the subsonic flow in the heliosheath [22]. Thus, the average upstream radial anisotropy should be the same as in the subsonic heliosheath even though the upstream wind is faster, meaning that 
the larger convective anisotropy must be substantially balanced by inward diffusion [22]. The observed magnetic-field strength indicates that Voyager 1 was in the fast solar wind upstream of the TS during this period [23].

There are two major features of the V1 TSR increases that are not yet understood.

(i) The peak of the ACR He intensity at this time is near $25 \mathrm{MeV} / \mathrm{n}$ compared to a peak energy of $6.5 \mathrm{MeV} / \mathrm{n}$ over the 1998 solar minimum-suggesting there is still significant modulation between V1 and the TS.

(ii) There are periods of very large anisotropies in the $\mathrm{MeV} \mathrm{H}$ ion data, which is predominantly field aligned, that flow in the direction away from the Sun.

It is expected that over the next several years there will be a continuing interplay between the increasing distance to the TS and the significant reductions expected in the modulation level as we move toward the solar minimum period of cycle 23 .

\section{REFERENCES}

1. Parker, E. N., Interplanetary Dynamical Processes, 115-128, (Interscience Division, John Wiley and Sons, New York, 1963).

2. Zank, G. P., Space Sci. Rev. 89, 413 (1999).

3. Jokipii, J. R., Kóta, J., and Merenyi, E., Astrophys. J. 405, $782-786$ (1993).

4. Steenberg, C. D., Ph.D. Thesis, Potchefstroom University C.H.E. (1998).

5. Fisk, L. A., Kozlovsky, B., and Ramaty, R., Astrophys. J. 190, L35, (1974).

6. Pesses, M. E., Jokipii, J. R., and Eichler, D., Astrophys. J. 246, L85-L88 (1981).

7. Gloeckler, G., et al., J. Geophys. Res. 99, 17637-17643 (1994).

8. Mewaldt, R., Proc. $24^{\text {th }}$ Int. Cosmic Ray Conf. (Rome) 3, 804-807 (1995).

9. Webber, W. R., and Lockwood, J. A., J. Geophys. Res. 106, 29,323 (2001).

10. Stone, E. C., et al., Space Sci. Rev. 21, 355 (1977).

11. Potgieter, M. S., and Ferreira, S. E. S., J. Geophys. Res. 107, A7, 10.1029/2001JA009040 (2002).

12. Webber, W. R., Simpson, G. A., and Cane, H. V., Astrophys. J. 236, 448 (1980).

13. Strong, A. W., et al., Astron. Astrophys. 292, 82 (1994).

14. Strong, A. W., Moskalenko, I. V., and Reimer, O., Astrophys. J. 537, 763 (2000).

15. Langner, V. W., de Jager, O. C., and Potgieter, M. S., Adv. Space Res. 27, 517 (2001).

16. Decker, R. B., McDonald, F. B., and Potgieter, M. S., Space Sci. Rev. 89, 278 (1999).

17. Krimigis, S. M., et al., Nature 426, 45 (2003).

18. McDonald, F. B., et. al., Nature 426, 48 (2003).

19. Cummings, A. C., et. al., Proc. $28^{\text {th }}$ Int. Cosmic Ray Conf. (Tsukuba) 7, 3777 (2003).

20. Wang, C., Richardson, J. D., and Burlaga, L. F., Solar Physics 204, 411 (2001).

21. Whang, Y. C., and Burlaga, L. F., Geophys. Res. Lett. 27, 1607 (2000).

22. Jokipii, J. R., and Giacalone, J., Astrophys. J. 605, L145 (2004).

23. Burlaga, L. F., et. al., Geophys. Res. Lett. 30, 20 (2003).

24. Huber, D. M., Ph.D. Thesis, Univ. of Delaware (1998).

25. Cummings, A. C., and Stone E. C., Adv. Space Res. 23, 509 (1999).

26. McDonald, F. B., et. al., J. Geophys. Res. 102, 4643 (1997). 